Márcia São Pedro Leal Souza'

Susan Martins Pereira"

Jamocyr Moura Marinho"'I

Maurício L Barreto"

\section{Características dos serviços de saúde associadas à adesão ao tratamento da tuberculose}

\section{Characteristics of healthcare services associated with adherence to tuberculosis treatment}

RESUMO

OBJETIVO: Analisar características relacionadas à adesão ao tratamento dos casos de tuberculose em serviços de referência para tuberculose.

MÉTODOS: Trata-se de um estudo ecológico nas unidades de referência no tratamento dos casos de tuberculose dos distritos sanitários de Salvador, BA, em 2006. A amostra foi composta pelas unidades de saúde municipais que atenderam $67,2 \%$ dos 2.283 casos notificados de tuberculose no ano. Foram analisadas as variáveis: cura, abandono, exames realizados, equipe de saúde e benefícios aos pacientes. Para verificar associação entre as variáveis, foi utilizado o teste qui-quadrado ou exato de Fisher, sendo consideradas estatisticamente significantes as associações com $\mathrm{p}<0,05$.

RESULTADOS: Dos casos estudados, 78,4\% resultaram em cura, 8,6\% em abandono, 2,2\% em óbito e 8,1\% em transferência. As taxas de adesão por unidade de saúde apresentaram variabilidade entre $66,7 \%$ a 98,1\%. As variáveis cura e abandono mostraram associação estatisticamente significante com a adesão na comparação de proporções. Todas as unidades com alta adesão possuíam equipe de saúde completa.

CONCLUSÕES: A adesão foi fator importante para o desfecho cura e abandono, mas foi baixo o índice de unidades que alcançaram as metas de cura. A presença de equipe multidisciplinar completa no programa de tuberculose pode contribuir para a compreensão pelo paciente sobre a sua enfermidade e a adesão ao tratamento para a cura.

Coletiva Instituto de Saúde Coletiva (ISC).

Universidade Federal da Bahia (UFBA).

Salvador, BA, Brasil

"ISC-UFBA. Salvador, BA, Brasil

II" Escola Bahiana de Medicina e Saúde Pública. Salvador, BA, Brasil

Correspondência | Correspondence: Márcia São Pedro Leal Souza

R. Basílio da Gama s/n

Campus Universitário do Canela UFBA

40110-040 Salvador, BA, Brasil

E-mail: marciaspls@yahoo.com.br
DESCRITORES: Tuberculose, prevenção \& controle. Pacientes Desistentes do Tratamento. Adesão ao Medicamento. Serviços de Saúde. Estudos Ecológicos. 


\section{ABSTRACT}

OBJECTIVE: To analyze characteristics related to adherence to tuberculosis treatment in tuberculosis outpatient clinics.

METHODS: An ecological study was conducted in outpatient clinics for the treatment of tuberculosis cases in the sanitary districts of Salvador, Northeastern Brazil, in 2006. The sample was composed of the municipal health units that assisted $67.2 \%$ of the 2,283 tuberculosis cases that were reported during the year. The following variables were analyzed: cure, dropout, tests, health team and benefits offered to the patients. Chi-square test or Fisher's exact test was used to verify the association between variables, and associations with $\mathrm{p}<0.05$ were considered to be statistically significant.

RESULTS: Of the studied cases, $78.4 \%$ resulted in cure, $8.6 \%$ in dropout, $2.2 \%$ in death and $8.1 \%$ in transference. Adherence rates per health unit varied between $66.7 \%$ and $98.1 \%$. The variables cure and dropout showed a statistically significant association with adherence in the comparison of proportions. All the units with high adherence rates had complete health teams.

CONCLUSIONS: Adherence was an important factor for the outcomes cure and dropout, but the index of units that achieved the cure goals was low. The presence of a complete multidisciplinary team in the tuberculosis program may help the patient understand his/her illness and contribute to adherence to treatment.

DESCRIPTORS: Tuberculosis, prevention \& control. Patient Dropouts. Medication Adherence. Health Services. Ecological Studies.

\section{INTRODUÇÃO}

A tuberculose (TB) é uma doença infecto-contagiosa, com evolução em ciclos lentos e maior incidência nas aglomerações urbanas. No Brasil, são notificados anualmente cerca de 85 mil casos novos, correspondendo a uma incidência de 47/100.000 habitantes. Ocorrem cerca de 6 mil óbitos decorrentes da doença (Sinan/MS). ${ }^{\text {a }}$ A partir de 2003, a TB foi inserida na agenda de prioridades das políticas públicas do Brasil. ${ }^{12}$ Em 2005, o País alcançou as metas intermediárias estabelecidas pela Organização Mundial da Saúde (OMS), com 73,5\% de detecção dos casos estimados e $69,3 \%$ de cura.

No Brasil, a estratégia do tratamento diretamente observado (DOTS) tem sido implantada em alguns serviços ligados ao Sistema Único de Saúde (SUS). No entanto, a escassez de recursos humanos e financeiros no sistema público de saúde representa uma dificuldade operacional em acompanhar os pacientes que realizam o tratamento de TB. ${ }^{2}$ Os primeiros dois a três meses de tratamento são os períodos nos quais ocorrem a maioria dos abandonos, indicando a necessidade de medidas que o reduzam desde o início do tratamento. ${ }^{7}$
Um ponto de discussão entre os profissionais de saúde tem sido a não-adesão ao tratamento medicamentoso. Entende-se que a adesão depende do sucesso da terapia proposta, da cura de determinada enfermidade e do controle de um processo crônico. Ao tomar como exemplo as pessoas que vivem com HIV/Aids, a OMS define adesão como um processo dinâmico, multifatorial, que envolve aspectos comportamentais, psíquicos e sociais e que requer decisões compartilhadas e co-responsabilidades entre usuário do serviço, a equipe de saúde e a rede social de apoio e com abordagem que atenda às singularidades socioculturais e subjetivas, objetivando uma melhor qualidade de vida das pessoas. ${ }^{14,15}$

Deste modo, a adesão ao tratamento pode ser baseada em abordagens individuais relacionados ao paciente ${ }^{8}$ ou fatores ambientais externos ao paciente. Os primeiros já estão bem descritos na literatura, a exemplo de pertencer ao sexo masculino, baixa escolaridade e tabagismo, uso de álcool ou drogas. ${ }^{2,11}$ Entretanto, há escassez de estudos sobre as características dos serviços de saúde e sua relação com adesão ao tratamento da TB. 
A cidade de Salvador, BA, é considerada prioritária no controle da TB pelo Ministério da Saúde (MS) por ser a terceira capital do País em números de casos notificados. São em média três mil casos-ano e incidência de 92,4/100mil hab em 2006, correspondendo a quase o dobro da incidência nacional de 48/100.000 hab (COAPS/SMS/2007ª). Para enfrentar este problema foi desenvolvido o Plano Municipal de Controle da Tuberculose (PMCT) com o objetivo de implementar as medidas de controle da TB como a detecção de casos novos, tratamento e cura.

O objetivo do presente estudo foi analisar características relacionadas à adesão ao tratamento dos casos de tuberculose em serviços de referência para tuberculose.

\section{MÉTODOS}

Trata-se de um estudo ecológico, transversal, descritivo, realizado nas unidades de referência para o tratamento dos casos de TB dos distritos sanitários no município de Salvador, em 2006.

A estimativa da população em Salvador, em 2005, era de 2.711.372 habitantes. ${ }^{\mathrm{b}}$ Nesse município há 12 distritos sanitários, 86 unidades básicas de saúde municipais, 63 unidades de saúde municipais com PCT e 41 unidades de saúde da família. Para o ano de 2006, foram notificados 2.283 casos de TB. Foram avaliados 11 distritos sanitários, representados por uma unidade de saúde por distrito, as quais são consideradas de referência para o controle de TB. As unidades incluídas atenderam a $67,2 \%$ do total de casos ocorridos em Salvador.

Inicialmente, foi listado o número de casos de TB em cada distrito sanitário e foram identificadas as unidades de saúde responsáveis pelo maior número de atendimento de casos em cada distrito, as quais foram incluídas no estudo.

Foi elaborado um questionário padronizado previamente testado para aplicação aos coordenadores do programa de controle da TB nos serviços.

Este instrumento incluiu questões sobre a identificação do distrito sanitário, oferta e qualidade da assistência à saúde e condições organizacionais dos recursos humanos da unidade de saúde.

Uma equipe composta de quatro alunos universitários foi treinada para coleta de dados, que ocorreu durante oito semanas. Dados secundários foram obtidos do livro de registro e controle de tratamento dos casos de tuberculose, disponível na unidade básica de saúde, para obtenção dos indicadores gerais das ações de controle dos casos de TB nas unidades de saúde e os respectivos desfechos em 2006.

Foi considerado como desfecho a adesão ao programa de tratamento da TB, expressa pelo percentual de adesão. As co-variáveis foram obtidas a partir dos aspectos relacionados às características dos serviços de saúde e do programa de controle de TB.

Para o cálculo da taxa de adesão, o numerador foi composto pela somatória do número de casos de cura comprovada, cura não comprovada e falência e o denominador considerou a população inscrita no programa de controle de TB (somatório do número de casos incidentes e em retratamento em cada unidade). Os casos de transferência foram descartados, uma vez que não seria possível obter dados sobre a permanência do paciente no programa. Os casos de falência permaneceram no cálculo da taxa de adesão, pois o MS define casos de falência como administração ao paciente dos três esquemas preconizados sem resposta ao tratamento. $\mathrm{Na}$ análise dos dados optou-se pela categorização dos percentuais da taxa de adesão em dois grupos: adesão inferior a $85,0 \%$ e adesão igual ou superior a $85,0 \%$. Este ponto de corte foi baseado no parâmetro de cura aceitável pelo Programa Nacional de Tuberculose, ${ }^{c}$ tendo em vista a inexistência na literatura de um parâmetro para este indicador.

Foi desenvolvido um escore para classificar as unidades básicas de saúde, baseado em características do serviço e das ações oferecidas pelo programa. $\mathrm{O}$ valor 0 foi considerado quando as características não estavam presentes na unidade; o valor 1 foi atribuído quando a realização do exame radiológico era feito na própria unidade, o valor 2 quando os benefícios (vale-refeição, Programa Bem Nutrir ${ }^{d}$ e vale-transporte) e ações de vigilância (busca ativa, busca de faltosos e veículo na unidade) estavam presentes na unidade e não ocorria falta de medicamento na unidade. Valor 3 foi atribuído quando a baciloscopia era realizada na própria unidade e para a presença de cada profissional na composição da equipe de saúde. Os valores utilizados para a pontuação das variáveis foram atribuídos a partir da relevância para o desenvolvimento dos objetivos do Programa Nacional de Tuberculose de acordo com as normas estabelecidas pelo MS, a exemplo de equipe de

\footnotetext{
a Secretaria Municipal de Saúde. Coordenadoria de Atenção e Promoção a Saúde. Dados atualizados em 12/11/2007. Disponível em DEF=C:/ SinanNet/BaseDBF/TuberculNET.def (Documento fornecido pelo próprio setor).

b Instituto Brasileiro de Geografia e Estatística. IBGE Cidades@. [citado 2009 out 22] Disponível em: http://www.ibge.gov.br/cidadesat/ topwindow.htm

c Comitê Técnico-Científico de Assessoramento à Tuberculose e Comitê Assessor para Co-infecção HIV-Tuberculose. Tuberculose: guia de vigilância epidemiológica. Brasília: Ministério da Saúde/Fundação Nacional de Saúde, 2002.

d O Programa Bem Nutrir, implantado em 1999, tem por objetivo fornecer suplemento alimentar no combate à tuberculose, constituído de pão e leite de soja a entidades sociais.
} 
saúde completa e realização da baciloscopia na própria unidade. As variáveis foram agrupadas de acordo com os aspectos diagnósticos, equipe, benefícios, vigilância e medicamento para verificação da associação das mesmas em relação à adesão. $\mathrm{O}$ escore obtido variou de 0 a 33 pontos.

Para análise, as variáveis foram agrupadas em relação a: realização de exames diagnósticos, equipe, benefícios, ações de vigilância e oferta de medicamentos. A primeira foi composta por serviço de radiologia em funcionamento na unidade e realização de baciloscopia na própria unidade. A variável equipe foi considerada como presença de médico, enfermeiro, auxiliar de enfermagem, assistente social e visitador do programa. Foram consideradas equipe completa as unidades que apresentaram todos os profissionais e equipe incompleta a ausência de um dos profissionais. A variável benefícios foi composta pela oferta do ticket refeição, do Programa Bem Nutrir e do vale transporte, sendo completa quando da presença de todos os benefícios e incompleta na ausência de pelo menos um dos itens. A variável vigilância foi composta pela busca ativa, busca dos faltosos e presença de veículo na unidade, sendo considerada a realização das ações. A variável medicamento foi determinada pela falta ou não do medicamento específico nos últimos seis meses.

Os dados foram analisados por meio do software Epi Info versão 6.04 e Stata 7.0. Foi realizada análise descritiva, variáveis contínuas foram analisadas utilizando-se medida de tendência central e dispersão e as variáveis categóricas como proporção. Para analisar a associação entre as variáveis nominais, foi utilizado o teste do qui-quadrado ou exato de Fisher. Foram consideradas associações estatisticamente significantes aquelas com $\mathrm{p}<0,05$.

O estudo foi aprovado pelo Comitê de Ética da Santa Casa de Misericórdia da Bahia. Os participantes assinaram termo de consentimento livre esclarecido e tiveram assegurado seu direito de participação voluntária, recusa e confidencialidade das informações, conforme Resolução 196/96 do Conselho Nacional de Saúde.

\section{RESULTADOS}

Considerando o total de unidades com PCT em Salvador, houve $69,9 \%$ de cura, enquanto que as unidades de referência apresentaram 78,4\%. Com relação ao abandono, Salvador apresentou 6,5\% e as unidades de referência $8,60 \%$. Em relação ao óbito, ocorreu $0,04 \%$ em Salvador e 2,2\% nas unidades. No desfecho transferência, a cidade de Salvador apresentou $13,3 \%$ e as unidades de referência 8,08\%. As diferenças encontradas entre o total de unidades em Salvador e unidades de referência em todos os desfechos foram estatisticamente significantes (Tabela 1).

Ao analisar a adesão por unidade de saúde, houve variabilidade nas taxas, sendo a menor de $66,7 \%$ na UBS 1, seguida da UBS 7 com 77,1\%. A maior taxa de adesão encontra-se na UBS 6 com 98,1\%, seguido da UBS 8, com 92,2\%.

Em relação ao desfecho cura, a UBS 1 apresentou o menor percentual $(66,7 \%)$, seguido da UBS 7 com $77,1 \%$. A UBS 6 obteve o maior percentual de cura, $98,1 \%$, seguido da UBS 8 com $92,2 \%$. No desfecho abandono, a UBS 6 obteve menor (1,3\%) abandono. As maiores taxas estavam presentes na UBS 1 com 33,3\% e na UBS 7 com 19,7\% (Tabela 2).

As seguintes unidades foram classificadas com baixa adesão: UBS 1, 3, 5, 7, 9, com um total de 442 casos de TB em tratamento, obtendo $72,2 \%$ de cura e $18,3 \%$ de abandono. As unidades UBS 2, 4, 6, 8, 10 e 11 foram classificadas como alta adesão, com 1.092 casos de TB em tratamento, $81,0 \%$ de cura e $4,7 \%$ de abandono. Observou-se associação estatisticamente significante entre adesão e proporções de cura e abandono (Tabela 3).

O serviço de radiologia estava presente em apenas três unidades (UBS 5, 6 e 9). A realização da baciloscopia na própria unidade ocorreu com maior freqüência (UBS 2, 4, 5, 6, 7, 9 e 10). A equipe de saúde completa estava presente nas UBS 2, 4, 6, 8 e 11. Os benefícios completos estavam presentes nas UBS 5 e UBS 11 . Em relação à variável vigilância, apenas a UBS 1 não possuía veículo na unidade.

Tabela 1. Proporção de alta por cura, por abandono, por óbito e transferência de pacientes no município e nas unidades de referência. Salvador, BA, 2006.

\begin{tabular}{lccccc}
\hline Local & Casos de TB (n) & Cura \% & Abandono \% & Óbito \% & Transferência \% \\
\hline Salvador $^{\mathbf{a}}$ & 2.283 & 69,90 & 6,53 & 0,04 & 13,32 \\
Unidades de referência $^{b}$ & 1.534 & 78,42 & 8,60 & 2,15 & 8,08 \\
\hline
\end{tabular}

a Fonte: Sistema de Informação de Agravos de Notificação; Coordenadoria de Atenção e Promoção à Saúde/Secretaria Municipal de Saúde.

b Unidade de referência de cada distrito sanitário

$\mathrm{P}<0,05$ 
Tabela 2. Taxa de adesão, proporção de cura e abandono de acordo com a população adscrita das unidades de saúde, sem os casos de transferência, no programa de tratamento da tuberculose. Salvador, BA, 2006.

\begin{tabular}{|c|c|c|c|c|}
\hline Unidade de saúde ${ }^{\mathrm{a}}$ & População adscrita $(\mathrm{n})^{\mathbf{b}}$ & Adesão & Cura & Abandono \\
\hline UBS 1 & 72 & 66,7 & 66,7 & 33,3 \\
\hline UBS 2 & 138 & 87,7 & 87,2 & 4,5 \\
\hline UBS 3 & 136 & 80,9 & 80,5 & 17,3 \\
\hline UBS 4 & 349 & 89,7 & 89,6 & 4,3 \\
\hline UBS 5 & 100 & 83,0 & 83,0 & 15,0 \\
\hline UBS 6 & 155 & 98,1 & 98,1 & 1,3 \\
\hline UBS 7 & 61 & 77,1 & 77,1 & 19,7 \\
\hline UBS 8 & 77 & 92,2 & 92,2 & 6,5 \\
\hline UBS 9 & 42 & 81,0 & 81,0 & 16,7 \\
\hline UBS 10 & 165 & 91,5 & 91,5 & 7,9 \\
\hline UBS 11 & 100 & 86,0 & 85,9 & 10,1 \\
\hline
\end{tabular}

a Unidade de referência de cada distrito sanitário do município para o Programa de Controle da Tuberculose.

b Para o cálculo dos percentuais de cura e abandono foi retirado da população adscrita os casos de falência.

Houve falta de medicamento em quatro unidades (UBS $1,4,10$ e 11).

Na classificação das unidades por escore, observou-se que as UBS 2, 5 e 6 apresentaram os escores mais elevados ( 30 e 31 pontos) e as UBS 2 e 6 obtiveram elevada adesão $(87,7 \%$, e $98,16 \%)$, respectivamente. Estas apresentavam em comum a presença do visitador do programa na equipe de saúde; o qual não estava presente na equipe da UBS 5 que atingiu um percentual de adesão inferior (83,0\%), apesar de pertencer ao grupo com elevado escore.

Analisando-se as duas unidades que obtiveram escore com pontuação 28 , observou-se que a UBS 4 apresentou $89,7 \%$ de adesão enquanto a UBS $981,0 \%$. Na primeira o auxiliar de enfermagem estava presente na equipe de saúde, o que não ocorria na UBS 9. O escore 27 foi observado nas UBS 7, 8 e 11. Destas, a UBS 8 e 11 apresentaram adesão acima de $85,0 \%$. A UBS 7 apresentou um percentual menor $(77,05 \%)$, observando-se nesta unidade a ausência do auxiliar de enfermagem na equipe de saúde. A UBS 10 apresentou escore 25, seguida da UBS 3 com 24 pontos (Tabela 4).

Nas sete unidades que realizavam a baciloscopia no próprio local e tinham serviço de radiologia em funcionamento, 42,9\% apresentaram baixa adesão. A proporção de unidades com alta adesão que realizavam estes exames foi maior $(57,1 \%)$, entretanto esta diferença não apresentou significância estatística (Tabela 5).

Em relação à equipe de saúde completa, observou-se que $100 \%$ das unidades com alta adesão possuíam esta característica, com equipe formada pelo médico, enfermeiro, auxiliar de enfermagem, assistente social e visitador. Das unidades com baixa adesão, a maioria $(83,3 \%)$ não atendia a este requisito, sendo esta diferença estatisticamente significante. Quanto aos benefícios oferecidos pelo serviço, $50,0 \%$ das unidades com adesão alta e baixa ofereciam benefícios completos, não havendo diferenças entre os grupos. Também não foram encontradas diferenças estatisticamente significantes entre presença de vigilância e medicamentos (Tabela 5).

\section{DISCUSSÃO}

O presente estudo identificou baixo percentual de cura $(69,9 \%)$ e alto percentual $(6,5 \%)$ de abandono no município de Salvador no ano de 2006. As unidades de análise estudadas são representativas dos serviços de saúde do município que desenvolvem ações de controle de TB. Esses achados de maior percentual de cura, abandono e óbito e menor transferência encontrados

Tabela 3. Percentual dos desfechos de cura e abandono de acordo com as unidades de referência classificadas como alta e baixa adesão ao tratamento de tuberculose. Salvador, BA, 2006.

\begin{tabular}{lcccc}
\hline Adesão & $\mathrm{N}^{\circ}$ unidades & Casos $^{\mathbf{a}}$ & Cura & Abandono \\
\hline$<85,0 \%$ & 5 & 442 & 72,2 & 18,3 \\
$\geq 85,0 \%$ & 6 & 1092 & 81,0 & 4,7 \\
\hline
\end{tabular}

a Total de casos de tuberculose nas unidades de referência de cada distrito $p=0,001$ 
Tabela 4. Escores para classificação das unidades básicas de saúde, baseado nos aspectos de diagnósticos, equipe, benefícios, vigilância e fornecimento de medicamento. Salvador, BA, 2006.

\begin{tabular}{|c|c|c|c|c|c|c|c|c|c|c|c|}
\hline Variável & UBS1 & UBS2 & UBS3 & UBS4 & UBS5 & UBS6 & UBS7 & UBS8 & UBS9 & UBS10 & UBS11 \\
\hline Adesão (\%) & 66,7 & 87,7 & 80,9 & 89,7 & 83,0 & 98,1 & 77,1 & 92,2 & 81,0 & 91,5 & 86,00 \\
\hline \multicolumn{12}{|l|}{ Diagnóstico } \\
\hline Serviço de radiologia & 0 & 0 & 0 & 0 & 1 & 1 & 0 & 0 & 1 & 0 & 0 \\
\hline $\begin{array}{l}\text { Baciloscopia na própria } \\
\text { unidade }\end{array}$ & 0 & 3 & 0 & 3 & 3 & 3 & 3 & 0 & 3 & 3 & 0 \\
\hline \multicolumn{12}{|l|}{ Equipe } \\
\hline Enfermeiro & 3 & 3 & 3 & 3 & 3 & 3 & 3 & 3 & 3 & 3 & 3 \\
\hline Médico & 3 & 3 & 3 & 3 & 3 & 3 & 3 & 3 & 3 & 3 & 3 \\
\hline Auxiliar de enfermagem & 0 & 3 & 0 & 3 & 3 & 3 & 0 & 3 & 0 & 0 & 3 \\
\hline Assistente social & 3 & 3 & 3 & 3 & 3 & 3 & 3 & 3 & 3 & 3 & 3 \\
\hline Visitador do programa & 0 & 3 & 3 & 3 & 0 & 3 & 3 & 3 & 3 & 3 & 3 \\
\hline \multicolumn{12}{|l|}{ Benefícios } \\
\hline Vale-refeição & 2 & 2 & 2 & 2 & 2 & 2 & 2 & 2 & 2 & 2 & 2 \\
\hline Programa Bem Nutrir & 0 & 0 & 0 & 0 & 2 & 0 & 0 & 0 & 0 & 0 & 2 \\
\hline Vale transporte & 2 & 2 & 2 & 2 & 2 & 2 & 2 & 2 & 2 & 2 & 2 \\
\hline \multicolumn{12}{|l|}{ Vigilância } \\
\hline Busca ativa dos casos & 2 & 2 & 2 & 2 & 2 & 2 & 2 & 2 & 2 & 2 & 2 \\
\hline Busca dos faltosos & 2 & 2 & 2 & 2 & 2 & 2 & 2 & 2 & 2 & 2 & 2 \\
\hline Veículo na unidade & 0 & 2 & 2 & 2 & 2 & 2 & 2 & 2 & 2 & 2 & 2 \\
\hline Medicamento & 0 & 2 & 2 & 0 & 2 & 2 & 2 & 2 & 2 & 0 & 0 \\
\hline Escore & 17 & 30 & 24 & 28 & 30 & 31 & 27 & 27 & 28 & 25 & 27 \\
\hline
\end{tabular}

nas unidades eram de se esperar, haja vista que os dados foram coletados nas unidades de referência in loco, não havendo a possibilidade de duplicidade dos casos, devido às transferências oficiais ou espontâneas entre as unidades de saúde durante o tratamento, ou em tratamentos por recidiva após cura ou abandono.

O percentual alto de cura nas unidades de referência estudado é esperado, também, em virtude de as unidades concentrarem a maior quantidade de serviços de diagnose e terapia, possuírem equipes completas de profissionais e ser rara a ausência de medicamentos.

Os resultados encontrados apontam que, de uma forma geral, o Programa de Controle de Tuberculose, no ano de 2006, não atingiu as metas de cura especificadas. Em relação ao abandono foi observado um elevado percentual, uma vez que se aceita até 5,0\% deste desfecho. ${ }^{10}$ Isso indica a fragilidade dos serviços de saúde e do PCT no que diz respeito à adesão do paciente ao tratamento. O MS ratifica a prioridade nas ações contra a TB, colocando em prática um plano nacional que prioriza a qualificação dos recursos humanos, a mobilização social, a qualificação da rede de laboratórios e o monitoramento e avaliação, esperando que haja um fortalecimento das ações, reduzindo o número de casos de TB na população e nos grupos vulneráveis.

Em relação ao desfecho transferência, os dados coletados nas UBS no estudo apontam uma menor proporção $(8,1 \%)$ que a observada na cidade de Salvador $(13,3 \%)$. O percentual alto de transferência contribui para a melhoria do desfecho de abandono e piora do desfecho cura.

Já em relação ao óbito observou-se que as UBS, referência no tratamento de $\mathrm{TB}$, apresentaram uma proporção superior aos dados de Salvador, provavelmente, em decorrência da coleta in loco. Embora todos os municípios brasileiros enviem suas notificações ao SINAN, a real entrada de dados informatizados ocorre em cerca de apenas $70 \% .{ }^{3}$ Um estudo realizado sobre vigilância epidemiológica e o SINAN mostrou que em 2003, um em cada quatro municípios brasileiros apresentava deficiência em captar casos novos de TB ou de registrar adequadamente os dados de notificação e acompanhamento. ${ }^{4}$

Um dos principais achados do presente estudo refere-se ao alcance do percentual de adesão nas unidades que possuíam equipe de saúde completa. Mesmo nas unidades com elevado escore, a presença do visitador do programa ou do auxiliar de enfermagem parecem contribuir com a alta adesão. Santos ${ }^{12}$ enfatiza a presença da equipe de saúde completa como de extrema relevância no serviço, uma vez que as estratégias do PNCT devem, preferencialmente, ser desenvolvidas por equipes multiprofissionais com objetivo de inclusão social do paciente, sem, entretanto esquecer a importância da 
Tabela 5. Características dos serviços de saúde de acordo com adesão ao programa de controle de tuberculose nas unidades de referência. Salvador, BA, 2006.

\begin{tabular}{|c|c|c|c|c|}
\hline Características do serviço & $\mathrm{n}$ & Adesão < 85,0\% & Adesão $\geq 85,0 \%$ & $\mathrm{p}<0,05$ \\
\hline \multicolumn{5}{|l|}{ Exames diagnósticos ${ }^{\mathbf{a}}$} \\
\hline Faz & 7 & 42,7 & 57,1 & \\
\hline Não faz & 4 & 50,0 & 50,0 & 1,00 \\
\hline \multicolumn{5}{|l|}{ Equipe $^{\mathbf{b}}$} \\
\hline Completa & 5 & - & 100,0 & \\
\hline Incompleta & 6 & 83,3 & 16,7 & 0,01 \\
\hline \multicolumn{5}{|l|}{ Benefícios ${ }^{c}$} \\
\hline Completo & 2 & 50,0 & 50,0 & \\
\hline Incompleto & 9 & 44,4 & 55,6 & 1,00 \\
\hline \multicolumn{5}{|l|}{ Vigilância $^{d}$} \\
\hline Faz & 10 & 40,0 & 60,0 & \\
\hline Não faz & 1 & 100,0 & - & \\
\hline \multicolumn{5}{|l|}{ Medicamento } \\
\hline Faltou & 4 & 25,0 & 75,0 & \\
\hline Não faltou & 7 & 57,1 & 42,7 & 0,54 \\
\hline
\end{tabular}

a Exames diagnósticos: serviço de radiologia e realização de baciloscopia na unidade.

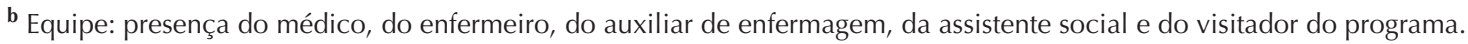

c Benefícios: ticket refeição, programa bem nutrir e vale transporte.

d Vigilância: busca ativa dos casos, busca dos faltosos e veículo na unidade.

organização do serviço sob a estratégia do tratamento supervisionado. Em relação à medicação, o acesso vem sendo garantido com aquisição e distribuição nos quantitativos necessários - 100 mil pacientes/ano, incluindo casos de TB multiressistente. ${ }^{12}$

Estudo realizado no Sul da Etiópia constatou que $26,0 \%$ dos faltosos mencionaram "estar se sentindo bem" como a principal razão para interromper o tratamento. ${ }^{6,13}$ Neste momento faz-se necessário a presença da equipe de saúde na unidade, treinada e organizada para adotar medidas que possam aumentar a adesão. A informação é considerada como fator relevante para a adesão ao tratamento, uma vez que o desconhecimento quanto à possibilidade de cura pode influenciar no abandono., ${ }^{9,10}$

A presença da equipe de saúde completa reforça a qualidade e organização do serviço, podendo ser um fator que influencia na redução do abandono do tratamento., $2,5,10$

Deheinzelin et $\mathrm{al}^{5}$ descrevem como fatores relevantes para uma boa adesão o estreitamento da relação médicopaciente e melhor organização do atendimento, que produzem, por sua vez, redução no tempo de espera para a consulta e treinamento dos médicos na avaliação e diagnóstico em TB.

No presente estudo, observou-se que todas as unidades de referência fornecem ao paciente, inserido no programa, o vale-transporte, possibilitando o seu deslocamento para o centro de saúde. Todavia, Paixão \& Gontijo ${ }^{10}$ não apontam a distância do serviço de saúde como fator relevante à não-adesão, uma vez que foi observada a preferência de alguns indivíduos em se tratarem longe do domicílio, por sentirem-se mais confortáveis, em virtude ao estigma da doença.

A queda na taxa de adesão produz redução no percentual de cura e conseqüente aumento no percentual de abandono de tratamento, apresentando relação direta não apenas com a disseminação da doença, mas também com o aparecimento das cepas multirresistentes. ${ }^{1,14}$

A alta adesão ao tratamento, conseqüentemente, com altos percentuais de cura, funciona como marcador da qualidade do serviço prestado na unidade, o que traduz o cumprimento do protocolo e o nível de competência da equipe de saúde.

Esperava-se no presente estudo que as UBS de referência para o tratamento da TB fossem contempladas com a presença de todas as características investigadas, obtendo pontuação máxima no escore. No entanto as UBS atingiram escores que variaram de 17 a 31 pontos, evidenciando algumas fragilidades no desenvolvimento das ações.

Consideramos que a possibilidade de viés de seleção pela inclusão das 11 UBS é pouco provável tendo em vista que estas são as unidades do Município mais representativas, concentrando o atendimento de maior 
número de casos de TB. Estas UBS são referências de cada distrito sanitário do município de Salvador em 2006, representando 67,2\% dos casos de TB. Apesar da possibilidade de algum viés de informação pelo fato das entrevistas terem sido realizadas junto aos supervisores do PNCT, os achados apontam variação entre as diversas unidades, de forma consistente com os desfechos apresentados.

\section{REFERÊNCIAS}

1. Alvarez-Gordillo GC, Alvarez-Gordillo JF, DorantesJiménez JE, Halperin-Frisch D. Percepciones y práticas relacionadas com la tuberculosis y la adherencia al tratamiento em Chiapas, México. Salud Publica Mexico. 2000;42(6):520-8. DOI:10.1590/S003636342000000600008

2. Bergel FS, Gouveia N. Retornos freqüentes como nova estratégia para a adesão ao tratamento de tuberculose. Rev Saude Publica. 2005;39(6):898-905.DOI:10.1590/ S0034-89102005000600005

3. Bierrenbach AL, Stevens AP, Gomes ABF, Noronha $E F$, Glatt R, Carvalho CN, et al. Efeito da remoção de notificações repetidas sobre a incidência da tuberculose no Brasil. Rev Saude Publica. 2007;41(Supl 1):67-76. DOI:10.1590/S0034-89102007000800010

4. Braga JU. Vigilância epidemiológica e o sistema de informação da tuberculose no Brasil, 2001-2003. Rev Saude Publica. 2007;41(Supl 1):77-88. DOI:10.1590/ S0034-89102007000800011

5. Deheinzelin D, Takagaki TY, Sartori AMC, Leite OHM, Amato Neto V, Carvalho CRR. Fatores preditivos de abandono de tratamento por pacientes com tuberculose. Rev Hosp Clin Fac Med Sao Paulo. 1996;51(4):131-5.

6. Kaona FAD, Tuba M, Siziya S, Sikaona L. An assessment of factors contributing to treatment adherence and knowledge of TB transmission among patients on TB treatment. BMC Public Health. 2004;4:68. DOI:10.1186/1471-2458-4-68

7. Menzies R, Rocher J, Vissandjee B. Factors associated with compliance in treatment of tuberculosis. Tuber Lung Dis. 1993;74(1):32-7. DOI:10.1016/09628479(93)90066-7
Em conclusão, a adesão é fator importante para o desfecho cura e abandono. Os resultados mostram que $54,6 \%$ das unidades do município de Salvador no ano avaliado alcançaram as metas estabelecidas para o desfecho cura, índice considerado baixo. A presença de equipe multidisciplinar completa no PCT pode contribuir para a compreensão do paciente sobre a sua enfermidade e a importância da adesão ao tratamento para a obtenção da cura.

8. Munro AS, Lewin SA, Smith HJ, Engel ME, Fretheim A, Volmink J. Patient Adherence to Tuberculosis Treatment: a systematic review of qualitative research. Plos Med. 2007;4(7):238. DOI:10.1371/journal.pmed.0040238

9. Oliveira HB, Moreira Filho DC. Abandono de tratamento e recidiva da tuberculose: aspectos de episódios prévios, Campinas, SP, Brasil, 1993-1994. Rev Saude Publica. 2000;34(5):437-43. DOI:10.1590/ S0034-89102000000500002

10. Paixão LMM, Gontijo ED. Perfil de casos de tuberculose notificados e fatores associados ao abandono, Belo Horizonte, MG. Rev Saude Publica. 2007;41(2):205-13. DOI:10.1590/S003489102007000200006

11. Rosemberg J. Tuberculose, aspectos históricos, realidades, seu romanticismo e transculturação. Bol Pneum Sanit. 1999;7(2):5-29.

12. Santos J. Resposta brasileira ao controle da tuberculose. Rev Saude Publica. 2007;41(Supl 1):8994. DOI:10.1590/S0034-89102007000800012

13. Shargie EB, Lindtjorn B. Determinants of treatment adherence among smear-positive pulmonary tuberculosis patients in Southern Ethiopia. Plos Med. 2007;4(2):37. DOI:10.1371/journal.pmed.0040037

14. World Health Organization. Adherence to long-term therapies: evidence for action. Tuberculosis. Geneva; 2003.

15. World Health Organization. Implementing the WHO Stop TB Strategy: a handbook for national tuberculosis control programmes. Geneva; 2008. (WHO/HTM/ TB/2008.401).

Artigo baseado na dissertação de mestrado de Souza MSPL, apresentada ao Programa de Pós-Graduação em Saúde do Instituto de Saúde Coletiva, Universidade Federal da Bahia, em 2008.

Pesquisa financiada pelo Conselho Nacional de Desenvolvimento Científico e Tecnológico (CNPq - Processo n478052/2004-9). 